Relations industrielles

Industrial Relations

\title{
L'Avenir professionnel des jeunes du milieu populaire, Pierre Idiart, Reine Goldstein, Collection " Jeunesse actualité ». Les Éditions Ouvrières, Paris, 1965. 205 pages.
}

\section{Gaston Ouellet}

\section{Volume 21, numéro 3, 1966}

URI : https://id.erudit.org/iderudit/027714ar

DOI : https://doi.org/10.7202/027714ar

Aller au sommaire du numéro

Éditeur(s)

Département des relations industrielles de l'Université Laval

ISSN

0034-379X (imprimé)

1703-8138 (numérique)

Découvrir la revue

Citer ce compte rendu

Ouellet, G. (1966). Compte rendu de [L'Avenir professionnel des jeunes du milieu populaire, Pierre Idiart, Reine Goldstein, Collection " Jeunesse actualité ». Les Éditions Ouvrières, Paris, 1965. 205 pages.] Relations industrielles / Industrial Relations, 21(3), 464-465. https://doi.org/10.7202/027714ar

Tous droits réservés (C) Département des relations industrielles de l'Université Laval, 1966
Ce document est protégé par la loi sur le droit d'auteur. L'utilisation des services d'Érudit (y compris la reproduction) est assujettie à sa politique d'utilisation que vous pouvez consulter en ligne.

https://apropos.erudit.org/fr/usagers/politique-dutilisation/ 
ployées. Hays suggère de les laisser exclusivement à l'action volontaire des parties. Quont aux problèmes des pressions exercées sur les arbitres relativement à leur réengagement futur por les parties, l'auteur rejoint les partisans des tribunaux du travail.

\section{Emile VALLeE}

Employment Policy and the Labor Market, Edited by Arthur M. Ross, University of California Press, 1965, 406 pages

Cet ourrage traite d'un sujet dont l'incidence sur la vie économique de toute nation industrielle est primordiale. Le chômage permanent, aux Etats-Unis, exige l'élaboration de politiques nouvelles, tant publiques que privées. C'est pourquoi le thème général du volume est qu'une compréhension profonde de la situation résultera en des politiques d'action efficaces à l'échelle nationale.

Cette recherche de solutions nouvelles à un vieux problème s'accompagne d'une critique objective de la relation entre le plein emploi et les autres buts de la politique économique américaine. Parmi ces derniers, on trouve la croissance économique, la stabilité des prix, la restriction des salaires, la balance des paiements, la restriction des dépenses fédérales et un budget fédéral équilibré.

11 est utile de mentionner que ce livre consiste en quatorze communications qui furent présentées à la Conférence sur le chômage à Boulder, Colorado, entre le 15 et le 19 juin 1964. Cette conférence s'inscrit dans le cadre d'un programme de recherche sur le chômage conduit par: « The Institute of Industrial Relations, University of California, Berkeley $»$. La première partie du volume porte sur les politiques générales d'emploi et établit une relation entre le chômage et l'inflation. Le modèle de R.A. Gordon sur les relations entre les diverses politiques, de même que la théorie des politiques de salaires-prix présentée par Murray Edelman et Robben W. Fleming, sont des apports importants à l'étude du chômage.

La seconde partie traite des causes, des concepts et des mesures du chômage. Au point de départ, on étudie la participation de la force de travail à l'aide d'une analyse statistique qui nous est fournie par William $G$. Bowen et T.A. Finegan. Plusieurs recherches sur le chômage camouflé, sur l'emploi vacant, sur le chômage saisonnier et surtout structurel se regroupent ici.
La troisième section de ce volume envisage le marché du travail et les politiques de formation. On y retrouve une analyse des coûts et des bénéfices des programmes ce formation qui fut effectué par Gerald G. Somers, de même qu'un exposé substantiel sur l'apprentissage, sa nécessité et ses lacunes.

Enfin, ovec Paul Jacob on laisse le domaine théorique pour jeter un coup d'oeil sur les chômeurs eux-mêmes. II y parvint en les écoutant, en vivant avec eux et même en prétendant être un des leurs.

$\mathrm{Ce}$ volume nous livre les recherches les plus récentes dans le domaine complexe du marché du travail tant sur le plan de l'offre que sur celui de la demande. Les résultats de ces recherches jettent une lumière nouvelle sur le chômage que l'on considère comme le premier problème économique des Etats-Unis. II est évident que plusieurs des chapitres de ce volume seront cités et discutés dans les années à venir.

\section{Claude-R. TETU}

\section{L'Avenir professionnel des jeunes du milieu populaire, Pierre Idiart, Reine Goldstein, Collection « jeunesse actualité », Les Edi- tions Ouvrières, Paris, 1965. 205 pages.}

Cette étude fait partie d'une série d'enquêtes menées en France por la J.O.C. et la J.O.C.F. et dont le but était de mettre en lumière les multiples obstacles que rencontrent les jeunes du milieu populaire pour réussir leur vie ouvrière. $A$ la suite des 60,000 réponses fournies par les jeunes qui ont participé à l'enquête-référendum intitulée: «Stop à l'échec des jeunes ", les outeurs ont essayé de dégager les différents problèmes de l'orientation professionnelle, de la scolarisation, de l'apprentissage et de l'emploi des jeunes travailleurs.

Dans une première partie intitulée: «Stop à l'échec de quels jeunes?", les auteurs tentent de montrer les grands profils statistiques de la jeunesse ouvrière française. On étudie d'abord la proportion des jeunes par rapport à la population globale puis par rapport au milieu populaire; on essaie ensuite de voir comment se répartissent ces jeunes dans le milieu scolaire et le milieu du travail. Les auteurs traitent aussi des différentes situations conditionnant la vie de travail des jeunes: qualifications des jeunes travailleurs, répartition suivant ces qualifications ainsi que distribution des jeunes dans les entreprises. 
Ces études statistiques peuvent nous sensibiliser aux nombreux problèmes que rencontrent les jeunes sur le marché du travail. Dans une deuxième partie, les auteurs poussent plus loin leur analyse et essaient de voir * comment les jeunes eux-mêmes s'éprouvent au milieu des difficultés qu'ils affrontent». On se penche sur les attitudes au travail des jeunes ouvriers et sur la connaissance du métier futur que peuvent avoir les * scolaires ». Puis à partir de différents " éléments professionnels » se dégagent quatre "situations-types» de travail sur lesquelles l'âge, le sexe et le métier auront une incidence. Enfin, dans une troisième partie, on cherche à savoir comment les jeunes choisissent leur école ou leur travail, qu'estce qui les motive et quels éléments influencent leurs choix.

Dans l'ensemble l'ouvrage semble nous donner une vue objective des différents problèmes et situations auxquelles les jeunes du milieu populaire ont à faire face dans le monde du travail; il nous permet « d'approcher la réaction subjective des jeunes du milieu populaire à la situation qui leur est faite ou devant l'avenir qui leur est tracé-»

\section{Gaston OUELLET}

Social Deviance, Social Policy, Action and Research, Leslie T. Wilkins, Prentice-Hall Inc., Englewood Cliffs, New Jersey, 1965. 305 pages.

Ce volume apporte au lecteur une étude des liens qui existent entre la recherche, la politique et le travail social. Après avoir expliqué la nature d'une étude scientifique, les distinctions entre l'action et la recherche, I'auteur précise le rôle de la théorie.

II constate la différence de langages qui se trouve entre théorie et pratique, dans les sciences de I'homme; il fait remarquer que le monde, l'objet étudié, est le même, mais que la théorie ne peut tenir compte des composantes affectives et émotionnelles des hommes; elle doit aussi mettre de côté les répétitions, les redondances que rencontre le praticien. C'est pourquoi théorie et pratique, recherche et action adoptent des langages différents, en parlant d'un même monde.

L'auteur applique ces considérations ou concept de « déviation sociale». II discute des sources de difficultés de communications, entre sciences pures et appliquées et il donne des suggestions de simplification. Si son application porte seulement sur les comportements anormaux ou pathologiques des hom- mes, c'est qu'il considère tous les problèmes sociaux, comme fondamentalement semblables; ainsi en expliquant les relations théoriepratique, par rapport au crime, l'auteur estime apporter les éléments de solution aux autres difficultés de ce genre.

Leslie T. Wilkins est "Senior Adviser * à l'Institut des Nations Unies d'Asie et d'Extrême-Orient, Tokyo, Japon; il est de nationalité anglaise; il a reçu le "Francis Wood Memorial Prize » de la Société Statistique Royale.

\section{Jeanne MICHAUD}

\section{Crime and Its Treatment in Canada, W.T.}

McGrath, Ed. The Macmillan Company of Canada Ltd., Toronto, 1965. 509 pages.

L'année 1965 a été marquée par une prise de conscience progressive des problèmes suscités par la criminalité et le système pénal canadien. Aussi le gouvernement fédéral du Canada a créé, en avril 1965, une Commission d'enquête sur la pénalité. Un intérêt à ces questions a été également à I'origine de la publication du volume que nous présentons ici. Celui-ci réunit seize exposés d'auteurs différents, sur les principaux aspects du crime et de la réhabilitation du criminel, au Canada, soit: les rapports crime et société; le taux de la délinquance ; le rôle de la police; I'organisation et lé fonctionnement des Cours de Justice; les formes d'institutions pénitentiaires; la libération conditionnelle: sa préparation, son organisation, ses difficultés: les réformes pénales et correctionnelles; le traitement du criminel

De plus, ces études, pour la plupart, sont accompagnées d'une bibliographie. Les auteurs se recrutent parmi des psychiatres, des avocats, des travailleurs sociaux, etc.; les uns occupent des postes de direction dans les institutions pénitentiaires et policières; d'autres se retrouvent dans l'enseignement ou la recherche; d'autres jouent un rôle de thérapeute, auprès des criminels... Ainsi la variété de la formation et des occupations des auteurs et la diversité des aspects traités procurent au lecteur une vue générale sur l'état actuel du crime et du système pénal canadien. Ce livre permet enfin de faire un pas plus avant, vers une coordination, une synthèse des opinions et points de vue des diverses spécialités intéressées, à la lumière des développements récents des sciences de I'homme.

Jeanne MICHAUD 